\title{
Sharp neck injuries in suicidal intention
}

\author{
Damien Biétry $\cdot$ Aristomenis Exadaktylos • \\ Thomas Müller · Peter Zbären • Marco Caversaccio • \\ Andreas Arnold
}

Received: 21 November 2014/ Accepted: 22 December 2014/Published online: 28 December 2014

(C) Springer-Verlag Berlin Heidelberg 2014

\begin{abstract}
Sharp neck injuries in suicidal intention often present as serious emergency situations with the need for an immediate diagnosis and treatment. We report our study of the clinical evolution of this emergency condition. This study investigates the cases of sharp neck injuries in suicidal intention treated at our institution between 2000 and 2010. Patient records were collected in a retrospectively reviewed and analyzed database. The current literature was compared to our findings. We found 36 cases (10 female and 26 male). The neck injuries were superficial and profound in 16 and 20 patients, respectively. Twenty-two patients were seen by the Head and Neck surgeon. A surgical neck exploration was necessary in 19 cases. Tracheal, laryngeal, pharyngeal and vascular injuries were found in one, five, three and three cases, respectively. The hospital stay ranged from 1 to 47 days. All the patients underwent emergency psychiatric assessment and were subsequently referred for psychiatric treatment. One patient died in the emergency room from an additional arterial injury to the wrist. Sharp neck injuries in suicidal intention treated with an interdisciplinary medical, surgical and psychiatric emergency assessment and treatment have low mortality and morbidity.
\end{abstract}

D. Biétry $(\bowtie) \cdot$ P. Zbären · M. Caversaccio · A. Arnold

Department of Otorhinolaryngology, Head and Neck Surgery Inselspital, University of Bern, 3010 Bern, Switzerland e-mail: damien.bietry@insel.ch

\section{A. Exadaktylos}

Department of Emergency Medicine, Inselspital, University of Bern, 3010 Bern, Switzerland

T. Müller

University Hospital of Psychiatry, University of Bern,

3010 Bern, Switzerland
Keywords Neck injuries - Suicide - Stabbing · Cutting · Sharp injury

\section{Introduction}

Switzerland has one of the highest suicide rates in the world, with 18 cases per 100,000 habitants [1]. In Switzerland, mortality due to suicide is threefold higher than secondary traffic accidents [2].

Compared with other methods, suicide by stabbing/ cutting is less common than other methods (hanging, poisoning, firearms, drowning, falling from a height) [3]. In approximately one-third of the suicides with stab/cut injuries, the neck is affected [4].

There are 10-15 suicide attempts per successful suicide. There are more men than women committing suicide. However, regarding the number of suicide attempts, the reverse is true [5]. Psychiatric disorders are reported in $90 \%$ of the cases of suicide [6].

Sharp neck injuries in suicidal intention (SNISI) often present as life-threatening neck injuries, requiring immediate emergency treatment. Between an injury of the neck, for example, in a traffic accident and a SNISI, significant differences in the injury patterns exist. An explanation is that in SNISI, vital structures are intended to be damaged, and the blunt trauma component and shear force injuries are often absent. For a better understanding and more efficient diagnosis and treatment of SNISI cases, an isolated investigation of this condition seems to be necessary.

Studies on traumatic penetrating neck injuries exist and generally address the management of the tracheal injuries in the case of an unstable airway [7-10].

To the best of our knowledge, the available literature contains only one similar study from Germany, explicitly 
concerning general neck injuries in suicide attempts; however, the primary on-site treatment has not been addressed [11].

The aim of our study was to critically review our management of patients with SNISI by retrospectively analyzing the outcome of our cases between 2000 and 2010 with regard to the morbidity and mortality and to identify contributing factors.

\section{Materials and methods}

The study was performed in a tertiary referral center and main emergency institution of the area (population of two million) with an annual emergency patient volume of 38,000 and annual serious trauma volume of 1,000 .

A search in the electronic emergency database of a tertiary referral center using the diagnosis keywords "neck injury" and "suicide" revealed 41 records. From these, 36 cases with SNISI were identified and analyzed retrospectively.

The following data were collected (from: the emergency protocol, surgery report, discharge report, and the followup record in the outpatient clinic)

(a) Patient data (age, gender, somatic and psychiatric medical history, season of the year)

(b) Primary on-site therapy and transport to the hospital (on their own, police, ambulance, rescue helicopter)

(c) Emergency assessment and treatment

(d) Pattern of injury (affected anatomical structures), surgical management

(e) Morbidity and mortality

(f) Duration of hospitalization

Psychiatric disorders were classified into slight (personality disorder; neurotic, stress-related and somatoform disorders; mental and behavioral disorders due to psychoactive substance use) and severe (schizophrenia, schizotypal and delusional disorders; affective disorders).

We defined the cut as profound when it was deeper than the platysma.

The correlation between the severity of the psychiatric disorder, gender and depth of the SNISI was analyzed statistically with a Pearson's Chi square test using SPSS (IBM, Armonk, New York, USA). A two-sided $p$ value of $<0.05$ was assumed to be significant.

A telephone interview was conducted regarding the outcome of the profound SNISI patients. The following points were addressed: Muscle weakness, problems with shoulder mobility, hoarseness and dysphagia in the past and in the present, psychiatric therapy, and drug therapy.

The current literature was compared to our findings.
This study was approved by the local ethics commission (Reg. 05-10-11).

\section{Results}

\section{Patient data}

Among the 36 cases with SNISI, 10 were female and 26 were male. Sixteen cases were superficial (one patient had four cases of SNISI) and 20 cases had profound neck injuries. The mean age of the women and men was 44 years (range 14-78 years) and 38 years (range 18-82 years), respectively.

The hospitalization ranged from 1 to 47 days. The psychiatric assessments revealed a slight and severe psychiatric disorder in 13 and 22 cases, respectively. In 33 cases, a psychiatric disorder was known before the SNISI. Five patients were regularly consuming cannabis, four patients were alcohol abusers, four were multiple drug users and three had a serious somatic problem (hepatitis C, prostate cancer and metastatic intestinal adenocarcinoma).

Patients with a severe psychiatric disorder presented significantly more often with a profound SNISI $(p=0.011)$. No significant correlations were found between the gender and psychiatric disorder or the gender and depth of the SNISI.

The average number of suicide attempts by SNISI treated at our institution during the observation period in women and men was one per year (range 0-2) and 2.6 per year (range 0-7), respectively. Interestingly, an increasing number of SNISI was observed in men beginning in 2007 (Fig. 1): four cases in 2008, five cases in 2009 and seven cases in 2010. The number for women was constant.

The seasonal distribution of SNISI showed a peak in the winter (15 cases), while nine cases occurred in the spring, seven cases in the summer and five cases in the autumn.

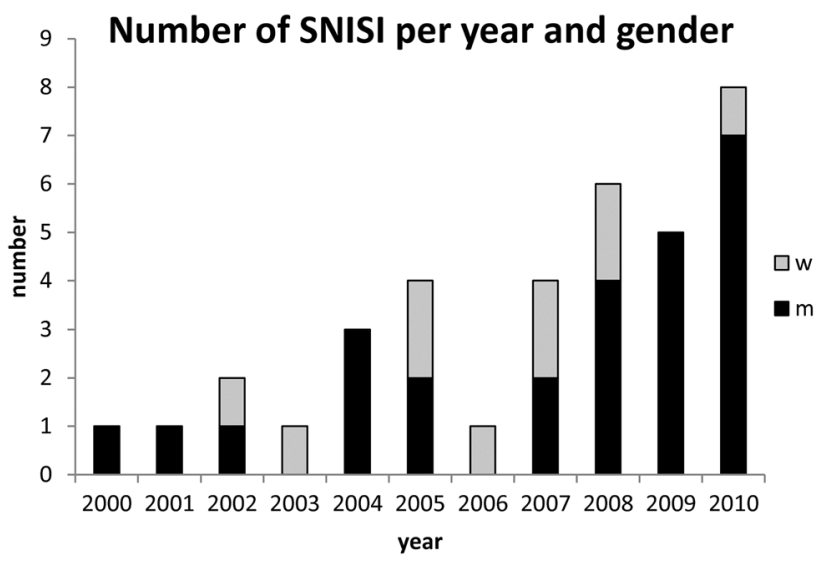

Fig. 1 Number of SNISIs per year and gender 
Primary on-site therapy and transport to the hospital

The patients with superficial injuries $(n=16)$ were transported by ambulance in $63 \%$ of the cases, by police in $25 \%$, and by helicopter in $6 \%$; $6 \%$ of the cases transported themselves.

The patients with profound injuries $(n=20)$ who were in cardiovascular and respiratory stable condition $(n=10)$ were transported by ambulance in $70 \%$ of the cases and by helicopter in $10 \%$; and $20 \%$ of the cases transported themselves (including one by bicycle).

The patients with profound injuries $(n=20)$ who were not in stable condition $(n=10)$ were transported by ambulance in $50 \%$ of the cases and by helicopter in $40 \%$, and $10 \%$ of the cases were transported by a family member. Five unstable patients $(50 \%)$ were intubated onsite by an ambulance team to stabilize their breathing.

\section{Emergency assessment and treatment}

In all 16 superficial SNISI cases, the surgical treatment was limited to wound disinfection and closure, which was performed by the emergency surgeon in 14 cases. In two patients, a Head and Neck surgeon was involved for the wound assessment because the SNISI was initially suspected to be profound.

In all 20 profound SNISI cases, a Head and Neck surgeon was involved. The first emergency assessment and treatment in the hospital was performed in the trauma room according to the Advanced Trauma Life Support (ATLS) guidelines [12]. The team leader was an emergency surgeon who called other specialists as required. The primary foci were the direct injuries to the airway, aspiration and hemorrhage. The priority was to stabilize the patient, in particular, to secure the airway.

The initially stable patients $(n=10)$ were assessed by the Head and Neck surgeon. Subsequently, further diagnostics or surgery was initiated. Three of these patients' cases had a computed tomography (CT) scan of the neck, one of which showed an airway injury (see Fig. 2). Two patients with suspected vascular injuries had a CT angiography (CTA) of the neck without any vascular injury.

Ten patients had insecure airways. As mentioned above, five of these were intubated on-site before transport. In the trauma emergency room, three were intubated and two underwent a tracheotomy. A bronchial lavage due to blood aspiration was necessary in one intubated patient. Four patients with suspected vascular injuries had a CTA without any vascular injury. Three had a CT scan of the neck that revealed an injury of the larynx in one case. One patient had a magnetic resonance angiography (MRA) to exclude a vessel or spinal cord injury; no esophagography was performed.
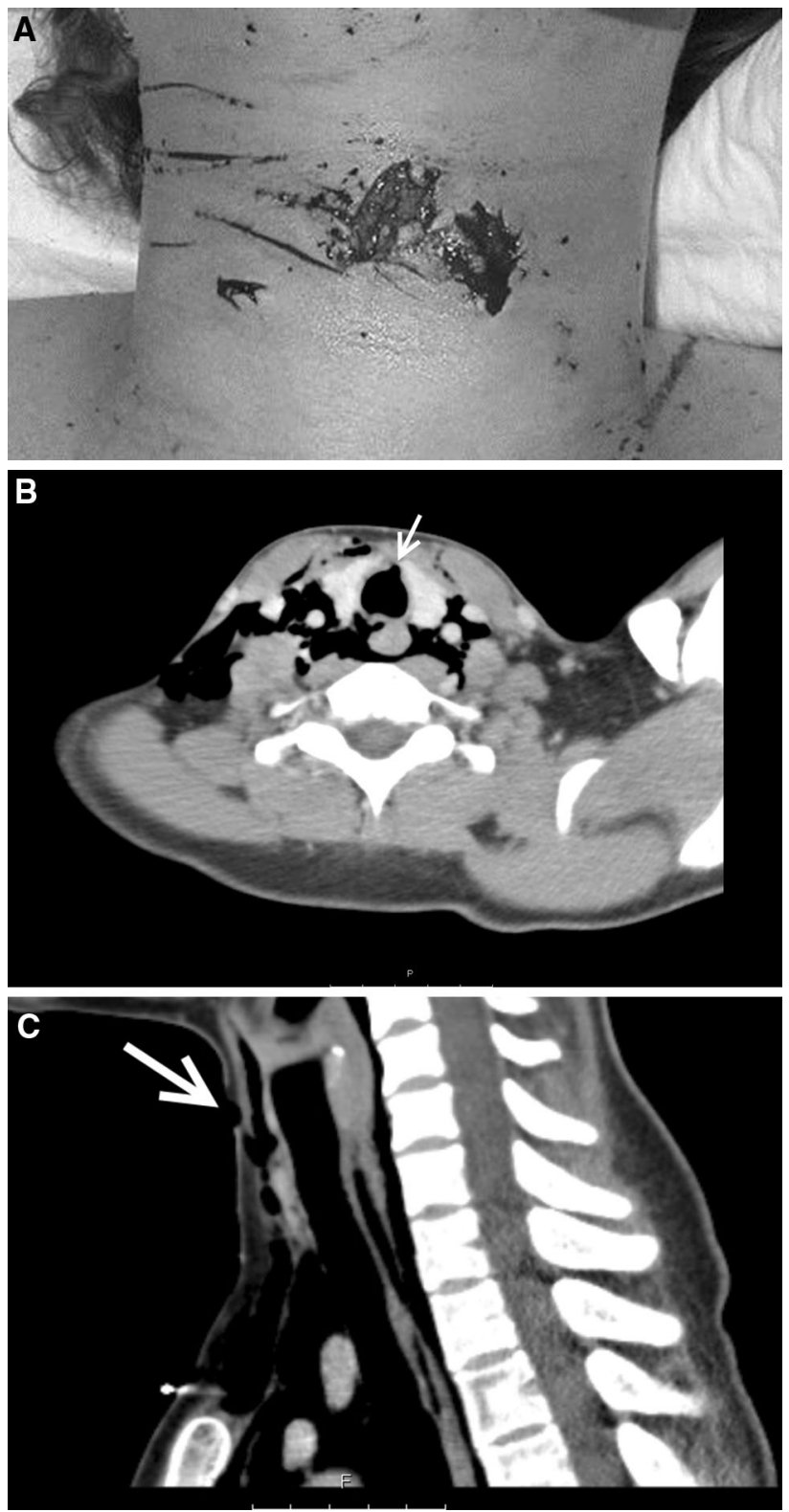

Fig. 2 Glass sharp SNISI. a Externally visible injury; b, c Axial and sagittal CT scan showing the airway injury (arrow) and resulting emphysema

The tetanus vaccination status was determined in all patients and was renewed if necessary.

Pattern of injury and surgical management

Superficial SNISI $(n=16)$

We defined a superficial SNISI as one that did not penetrate the platysma. The platysma is a layer that is easily identified. All the superficial SNISIs were closed primarily. 
One patient presented four times with superficial SNISIs after repeated suicide attempts but never had serious or profound injuries.

Nine patients with superficial SNISIs had additional injuries elsewhere that were treated by other specialists: five had superficial injuries of the extremities, two had thoracic injuries (one with a pericardial effusion) and two had abdominal injuries (one with a liver laceration).

The patients with superficial SNISIs had cut themselves with razor blades (7), scissors (3), knives (3), or a box cutter (1). In two patients, the method of cutting was unknown.

\section{Profound injuries $(n=20)$}

All the profound SNISIs were cutting injuries and not stab wound injuries. The injuries were inflicted with knives (15), a cardboard cutter (1), a glass shard (1), or a can lid (1). In two cases, the cutting instrument was unknown. Profound SNISIs were seen in $70 \%$ of the female and $50 \%$ of the male patients.

A common method of classification for penetrating neck injuries was described in 1979 by Roon and Christensen and divides the neck into three zones anterior to the sternocleidomastoid muscles. Zone I extends from the clavicles to the cricoid, zone II from the cricoid to the angle of the mandible, and zone III from the angle of the mandible to the skull base [13]. According to the aforementioned classification system, all of our patients had injuries in zone II. One cut affected both zones I and II.

A surgical neck exploration was performed in 19 cases because one patient died in the emergency room of exsanguination from a wrist injury.

Tracheal, laryngeal, pharyngeal and vascular injuries were found in one, five, three and three cases, respectively.

One interesting case with cuts from a glass shard (Fig. 2a) initially seemed to have been superficial. Only the CT scan (Fig. 2b, c) revealed air around the vessels resulting from an opened airway.

\section{Associated injuries}

In $50 \%(n=10)$ of the cases, we found wrist injuries on one or both sides, of which $40 \%$ were profound with injuries to tendons, nerves or arteries. Two patients presented direct thoracic injuries. The patient with SNISI in Zone I had emphysema in the mediastinum and an injury of the left thoracic aperture with a pneumothorax. One patient with a thoracic injury had a perforating injury of the abdominal wall without intra-abdominal involvement. Another patient with a perforating injury of the abdominal wall also presented without any intra-abdominal involvement but had additional injuries of the scrotal skin.

\section{Surgical management and further treatment}

Stabilization measures were performed in the emergency room. Nineteen patients with profound injuries (deeper than the platysma) underwent an exploration in the operating theater. Preoperatively, the patients received prophylactic antibiotics (mostly amoxicillin and clavulanic acid). The primary exploration of the SNISI focused on the airway and large vessels of the neck. The wound was cleaned and disinfected. In cases of suspected airway or pharyngoesophageal injury, we performed an endoscopy to assess the extent:

In $42 \%$ of patients (eight of 19) we performed a pharyngolaryngoscopy, in $11 \%$ (two of 19) a panendoscopy and in $26 \%$ (five of 19) the pharyngotracheal injury required a tracheotomy. In $47 \%$ of patients (nine of 19), a nasogastric feeding tube was placed because of an injury of the hypopharynx or prolonged intubation.

No esophageal injuries were found. Injuries of the larynx were reconstructed with absorbable sutures (Vicryl 3-0). After several days, oral feeding was initiated with clear liquids.

A psychiatric assessment of all the patients was undertaken as soon as possible. After recovering from their injuries, all the patients with a profound SNISI were hospitalized for psychiatric treatment. Of the patients with superficial injuries, 11 were hospitalized for psychiatric treatment; three were returned to prison, while the remaining two patients received outpatient psychiatric treatment according to the assessment of the psychiatrist.

\section{Morbidity and mortality}

Three patients suffered from pneumonia, two of whom had a profound neck injury that required the airway to be secured (one intubation, one tracheotomy). In one patient with superficial SNISI, an abdominal injury had to be explored. One patient with a laryngeal injury had postoperative hoarseness, which was successfully treated with speech therapy. The same patient developed a subcutaneous abscess around the sutures on the upper thorax, which was treated by an incision. Another patient with a hypopharyngeal injury had minor dysphagia that resolved spontaneously.

Another patient had decreased shoulder function that resolved after 6 weeks of physiotherapy. In one patient, a hypertrophic scar was excised.

None of the patients died due to a profound neck injury. One patient with a profound neck injury died in the emergency room; the autopsy found a lethal hemorrhage of a vessel injury on the wrist. 
Duration of hospitalization

The duration of hospitalization depended on the extent of the SNISI and also on injuries elsewhere on the body. The average stay was 7 days (range 1-47 days). The average stay of the patients with superficial SNISI and with or without wrist injuries was 3.7 days (range 1-8 days). In the patients with superficial SNISI and other body injuries, the average length of hospitalization was 16.6 days (range 5-28). In the patients with a profound SNISI and with or without wrist injuries, the average length of hospitalization was 6.3 days (range 1-18). For the patients with profound SNISI and other body injuries, the average length of hospitalization was 22.3 days (range 3-47 days).

Some patients were transferred to other hospitals, explaining their relatively short stay at our institution.

\section{Outcome}

A telephone interview was possible in nine of 19 patients with profound SNISI. The follow-up was between 4 months and 8 years (average $=43$ months). There were no complaints about any deficiencies in the neck region.

Four patients were still in outpatient psychiatric treatment and one patient is still hospitalized in a psychiatric institution. Five patients were able to stop psychotropic drug treatment.

\section{Discussion}

\section{Patient data}

In our study, many men (26) than women (10) presented with SNISI; this finding agrees with the report of Herzog et al. [11] that reflected all of the modes of injury in suicidal intention. It is typical for men to commit suicide in more aggressive ways, such as strangulation, shooting, cutting, and car accidents [3, 4]. In a study on the suicide attempts of 1,843 persons between 2004 and 2010 in the Bern agglomeration, $15.8 \%$ of suicide attempts (women $10.8 \%$, men $22.5 \%$ ) were from cutting injuries; this method was the second most frequent but had a low mortality. No increase in the rate of suicide attempts by cutting was reported during the studied period [3]. In contrast, we noted an increase for men during the final 6 years of our study period (Fig. 1).

In the report of Herzog et al. [11], only one of 27 patients from 1991 to 2002 was found with depression. In our study, 33 of 36 patients had a psychiatric history. It is known that a psychiatric disorder is an important risk factor for suicide attempts [14].
The number of suicide attempts was too small to detect a significant seasonal accumulation. Our peak was in winter and spring and the lowest point was in summer and autumn. Sebestyen et al. [15] noted a peak in spring/early summer and a low point in winter, which was discussed as a consequence of the seasonal incidence of depressionrelated suicides. The patients with a severe psychiatric disorder presented significantly more often with a profound SNISI ( $p=0.011)$, which were most often inflicted with a knife. Our results support the reports in the literature that severe psychiatric disorders, such as endogenous psychosis (including affective disorders and schizophrenia), cause more violent behavior in acts or attempts of suicide [16, 17]. In his classic report, Asberg et al. [18] described the correlation between the severity of the disease, the serotonin levels in the cerebrospinal fluid and the method of the suicidal act; the patients with a severe psychiatric disorder and low serotonin levels attempted suicide more often and used more violent means.

Primary therapy on field and transportation to the hospital

The study shows that the triage, first aid and transportation to a tertiary care center in our workflow are working well. There was no related mortality or morbidity.

Emergency assessment and treatment

In our study, the main life-threatening problem in SNISI was an injury to the upper airway. We found only small vessel injuries in the neck, without fatal hemorrhagic problems. Burgess et al. [19] formulated clinical guidelines for the assessment and management of a penetrating neck trauma. Furthermore, he recommends concentrating on the $\mathrm{C}$ of the $\mathrm{ABCDE}$ in the case of massive hemorrhage, thereby focusing on the article of Sarkar et al. [20] that describes the changing of the standard ATLS protocols from $\mathrm{ABCDE}$ to $\mathrm{CABDE}$ on the battlefield in assessing and controlling a hemorrhage which is the leading cause of morbidity and mortality. This focus prioritizes the circulation before returning to the traditional primary survey. On the contrary, we have good experience using the standard advanced trauma life support protocols in cases of SNISI.

Pattern of injury and surgical management

\section{Superficial injury}

In the group of patients with superficial SNISI, $56 \%$ (nine of 16) had other cutting injuries on the body, of which $44 \%$ (four of nine) were serious injuries. Mostly, the injuries were inflicted with a razor blade (7). 
Only one patient presented four times with superficial SNISIs after repeated suicide attempts but never had serious injuries. Five other patients (two with superficial and three with profound injuries) had a history of previous suicide attempts. It is known that a suicide attempt is an important risk factor for more suicide attempts-even 20 years after the first attempt [3].

\section{Profound injury}

The patients mostly cut themselves with knives (15), followed by a box cutter (1), glass shards (1), and a can lid (1). In two cases, the cutting instrument remained unknown. Interestingly, no profound SNISI was inflicted with a razor blade or scissors. Our findings are in accordance with the report of Karger et al. [21] which found that a knife is typically used for suicide attempts with SNISI.

In our study, a surgical exploration of the SNISI was performed if the injury was deeper than the platysma in 19 patients. In his article, Burgess et al. [19] found agreement in the literature for the need for surgical exploration in cases of "hard signs": a major airway injury (airway compromise, massive subcutaneous emphysema or a bubbling trough wound) or a major vascular injury (severe active bleeding, refractory shock, evolving stroke or a rapidly expanding hematoma). Thirteen of our 19 patients with profound SNISI presented with such hard signs.

Furthermore, Burgess reported the feasibility, based on several case series, of a conservative approach to the management of asymptomatic patients with a negative physical examination and the absence of findings on appropriate investigations in patients with penetrating neck trauma. The CTA may by the examination of choice for supposed arterial injuries [19]. Interestingly, one case in our series initially presented with a "hard sign" of an opened airway after cutting the thyrohyoid membrane but without any bleeding. The CTA did not show any vascular damage. During the surgical exploration, we found an unexpected injury to the superior thyroid artery.

\section{Morbidity and mortality}

Secondarily to SNISI, we found a short-term morbidity of $5 \%$ and a mortality of $0 \%$. One patient died secondarily to an arterial injury at the wrist. In a study by Roon and Christensen, the mortality in 189 patients with a penetrating neck trauma was $2.6 \%$. A routine exploration can minimize the morbidity of wounds penetrating the platysma [15].

A long-term outcome assessment was possible in nine of our patients, none of whom showed morbidity due to the SNISI.
Limitations of our study are the low number of patients despite a 10-year observation period, the difficult follow-up due to the retrospective nature of the study and the lack of comparison of our results to another emergency center.

\section{Conclusion}

Our patients who underwent emergency treatment for SNISI showed a good outcome with low morbidity and zero mortality related to the neck injury. It should be emphasized that the treatment of SNISI, included an interdisciplinary team of medical, surgical and psychiatric specialties. SNISIs are often accompanied by other injuries (e.g., to the thorax, abdomen, or extremities).

Although SNISIs often appear to be severe at first, lesions to the important vascular structures were rare. One reason may be the protected location behind the sternocleidomastoid muscle. Nevertheless, sharp injuries with clear borders can hide a more severe deep lesion and, therefore, merit exploration. In our experience, at least all of the wounds going deeper than the level of the platysma should be explored surgically.

Acknowledgments We thank Christian Wolf for his kind assistance in formatting the document and figures.

Conflict of interest The authors declare that they have no conflict of interest.

\section{References}

1. Suicide rates per 100,000 by country, year and sex (Table) [http:// www.who.int/mental_health/prevention/suicide_rates/en/]

2. Number of deaths and standardised mortality rates by age groups per 100,000 inhabitants (Table) [http://www.bfs.admin.ch/bfs/ portal/en/index/themen/14/02/04/key/01.html]

3. Reisch T, Steffen T, Maillart A, Michel K (2010) Suicide attempts in the county of Bern between 2004 and 2010: results from the WHO//MONSUE multicentre study on suicidal behaviour. In: Swiss Federal Office of Public Health FOPH

4. Fukube S, Hayashi T, Ishida Y, Kamon H, Kawaguchi M, Kimura A, Kondo T (2008) Retrospective study on suicidal cases by sharp force injuries. J Forensic Leg Med 15:163-167

5. Eberhardt MS, Ingram DD, Makuc DM (2001) Suicide. In: Urban and Rural Health Chartbook Health, United States, Hyattsville, Maryland, National Center for Health Statistics. p 58

6. Beautrais AL (2000) Risk factors for suicide and attempted suicide among young people. Aust N Z J Psychiatry 34:420-436

7. Brywczynski JJ, Barrett TW, Lyon JA, Cotton BA (2008) Management of penetrating neck injury in the emergency department: a structured literature review. Emerg Med J 25:711-715

8. Dupanovic M, Fox H, Kovac A (2010) Management of the airway in multitrauma. Curr Opin Anaesthesiol 23:276-282

9. Erhart J, Mousavi M, Vecsei V (2000) Penetrating injuries of the neck, injury pattern and diagnostic algorithm. Der Chirurg; Zeitschrift fur alle Gebiete der operativen Medizin 71: 1138-1143 
10. Maier H, Tisch M, Lorenz KJ, Danz B, Schramm A (2011) Penetrating injuries in the face and neck region. Diagnosis and treatment. HNO 59:765-782

11. Herzog M, Hoppe F, Baier G, Dieler R (2005) Injuries of the head and neck in suicidal intention. Laryngorhinootologie 84:176-181

12. American College of Surgeons Committee on Trauma (2004) Advanced trauma life support program for doctors, 7th edn. American College of Surgeons, Chicago

13. Roon AJ, Christensen N (1979) Evaluation and treatment of penetrating cervical injuries. J Trauma 19:391-397

14. Flavio M, Martin E, Pascal B, Stephanie C, Gabriela S, Merle K, Anita RR (2013) Suicide attempts in the county of Basel: results from the WHO/EURO multicentre study on suicidal behaviour. Swiss Med Wkly 143:w13759

15. Sebestyen B, Rihmer Z, Balint L, Szokontor N, Gonda X, Gyarmati B, Bodecs T, Sandor J (2010) Gender differences in antidepressant use-related seasonality change in suicide mortality in Hungary, 1998-2006. World J Biol Psychiatry 11:579-585
16. LA Dumais A, Lalovic A, Séguin M, Tousignant M, Chawky N, Turecki G (2005) Is violent method of suicide a behavioral marker of lifetime aggression? Am J Psychiatry 162:1375-1378

17. Beautrais AL (2002) A case control study of suicide and attempted suicide in older adults. Suicide Life Threat Behav $32: 1-9$

18. Asberg M, Traskman L, Thoren P (1976) 5-HIAA in the cerebrospinal fluid. A biochemical suicide predictor? Arch Gen Psychiatry 33:1193-1197

19. Burgess CA, Dale OT, Almeyda R, Corbridge RJ (2012) An evidence based review of the assessment and management of penetrating neck trauma. Clin Otolaryngol 37:44-52

20. Sarkar D, Demma A, Stulz D, Hsue G (2009) Expect the unexpected: two cases of penetrating head and neck trauma from Operation Iraqi Freedom. Ear Nose Throat J 88:E19-E21

21. Karger B, Niemeyer J, Brinkmann B (2000) Suicides by sharp force: typical and atypical features. Int J Legal Med 113:259-262 